\title{
Application of electric cell-substrate impedance sensing toward personalized anti-cancer therapeutic selection
}

\author{
Audrey F. Adcock ${ }^{1,2}$, Chiagozie O. Agbai ${ }^{1,2}$ and Liju Yang ${ }^{1,2^{*}}$
}

\begin{abstract}
Background: Cell-based analysis may have the potential for using patient-derived tissues/cells as a basis for a more direct prediction of therapeutic outcomes.

Methods: This study focused on the application of the Real Time Cell Electronic Sensing (RT-CES) system for screening effective anticancer drugs to different types of prostate cancers. The goal was to demonstrate the potential application of such technology toward the suitability in selection of personalized medicine. Three prostate cancer cell lines, DU145, LNCaP, and PC-3, and a normal prostate cell line were used. Docetaxel, Carboplatin, Abiraterone Acetate, Mitoxantrone, Sunitinib Malate were used as testing drugs.

Results: Cellular adhesion, proliferation and drug induced cellular responses were monitored by the RTCES system, and the results were correlated to the well-established cell viability tests. Identification of an effective drug from a panel of available anticancer drugs to a specific cancer cell line, or testing the effectiveness of a certain drug to a panel of prostate cancer cell lines was demonstrated. Cellular resistance to a drug at single dose and multiple dose challenges was monitored by RT-CES measurement, while such resistance was not detectable by endpoint cell viability assays.
\end{abstract}

Conclusions: The results of this study highlighted the advantages of such real-time impedance-based sensing system for applications in chemotherapy reagent selection.

Keywords: Cellular impedance-based sensing, Real-time cell-based assay, Metastatic prostate cancer, DU145, PC-3, LNCaP

\section{Background}

Personalized medicine is therapeutic treatments derived from diagnostic analysis of samples similar to that of the patient ((ASCO), American Society of Clinical Oncology 2017). The expectation is samples that are genetically or otherwise similar will respond in a similar manner to the same treatment. The most commonly used personalized medicine diagnostic tests are genetic based. The challenge presented by this method is genetic mutations do not specifically predict therapeutic outcomes (Hamburg and Collins 2010). Cell-based analysis may have the potential for

\footnotetext{
* Correspondence: lyang@nccu.edu

'Department of Pharmaceutical Sciences, Biomanufacturing Research Institute and Technology Enterprise (BRITE), North Carolina Central University, Durham, North Carolina 27707, USA

2Biomanufacturing Research Institute and Technology Enterprise (BRITE),

North Carolina Central University, 1801 Fayetteville Street, Durham, NC 27727, USA
}

using patient-derived tissues/cells as a basis for a more direct prediction of therapeutic outcomes. With this regard, cell-based biosensors, which use living cells or tissues as a sensing element to monitor physiological and functional changes induced by external stimuli, would be advantageous. Indeed, cell-based biosensors have become an important tool in the drug discovery process to provide a simple, fast, and cost-effective approach to screen compounds for drug candidates at very early stage and avoid unnecessary large-scale and cost-intensive animal testing (Asphahani and Zhang 2007; Cooper 2006; Liu et al. 2014; Zhang et al. 2012). Particularly, label-free and non-invasive cell-based assay/sensors are becoming of great interest in many biomedical applications (Fang 2006, 2011, 2014; Hu et al. 2013; Kim et al. 2009).

In recent years, a number of label-free technologies, including cell-substrate impedance (Giaever and Keese 
1993; Giaever and Keese 1986), quartz crystal microbalance (QCM) (Atay et al. 2016; Braunhut et al. 2005; Zhou et al. 2000), and optical waveguide lightmode spectroscopy (OWLS) (Fang 2007; Ramsden et al. 1995), have been reported as a means for monitoring live cell status in a non-invasive and real-time manner. The QCM biosensors sense the mass or surface property change upon the binding of biological targets, through the change in frequency of a quartz crystal resonator. The OWLS biosensors sense the absorption or binding of targets on the sensor surfaces through the change in optical signals. The electrical impedance cell-based biosensors have been recognized as a useful approach that is based on electrical signals, it enables direct analysis of cellular activities occurring on an electrode surface by measuring the induced capacitance and/or resistance changes by cells attached on the electrode, in a label-free and non-invasive manner. These changes have been associated with cell proliferation, spreading, motility, and death. Such impedance-based techniques have gained a great deal of attention in the fields of studying cancer cells and monitoring drug-induced cellular activities for drug discovery (Asphahani and Zhang 2007; Chen et al. 2008; Kloß et al. 2008; Linderholm et al. 2007; Liu et al. 2009; McGuinness 2007; Solly et al. 2004; Xiao and Luong 2003). Previously, our group has used such impedance-based technique to study oral cancer cells (Arias et al. 2010) and their responses to drug treatments, as well as to distinguish cancer cells and non-cancer cells (Yang et al. 2011). Another electrical method--field-effect transistors (FET)--has also been exploited in cell-based biosensors for real-time detection of targets. In the detection, either the concentration/activity of the target analyte or the presence/quantity of a biomolecule is transduced to an electrical signal via the field effect. Fundamental principles and various applications of FET biosensors have been extensively reviewed (Kaisti 2017).

This study focuses on the application of the impedance-based real-time cell analyzer (RTCA) system (ACEA Biosciences, Inc., San Diego, CA, USA) toward the selection of personalized anti-cancer medicine. Three established metastatic prostate cancer lines and a normal prostate cell line were used to demonstrate the rapid and real-time monitoring of cellular responses to five anticancer drugs, revealing the potential of such technology for selecting the effective drug for a given cell line and for testing a given drug to a panel of cell lines, as well as in special cases of monitoring drug resistance developed during treatment. The three tested prostate cancer cell lines included DU145, a brain metastatic PC cell line, LNCaP, a lymph node metastatic cell line, and PC-3, a bone metastatic PC cell line, and a normal prostate cell line. Docetaxel, the global gold standard for treating metastatic castration-resistant prostate cancer (mCRPC), was used as a testing drug (Hwang
2012). Other drugs included sunitinib malate, abiraterone acetate, mitoxantrone, and carboplatin.

\section{Methods \\ The RTCA impedance analyzer and cell index signal measurement}

The RTCA DP (Real-Time Cell Analyzer Dual Plate) Instrument (Roche and ACEA Biosciences, Inc., San Diego, CA, USA) was used in this study. The system is composed of a computer software control unit, an electronic sensor analyzer and three E-16-well plates. The computer control unit is used to set up the experimental parameters, collect the data from the analyzer in real time, and process the results. The electronic analyzer is to hold E-plates where the cells are seeded and growing and to execute the experimental protocol and record the electrical signals from the E-plates. The E-plates are single-use disposable plastic plates with an array of circle-on-line gold microelectrodes integrated into the bottom of the wells. The diameter of the circle is $100 \mu \mathrm{m}$. The electrode surfaces are pretreated for cell culture.

In operation, cells were seeded in the plates, and the plates were mounted to the sensor analyzer station. The electronic analyzer station was placed inside an incubator at $37{ }^{\circ} \mathrm{C}$, with $5 \% \mathrm{CO}_{2}$ in air. The system was connected to the computer control unit which was placed outside of the incubator. The system monitored cellular activities by measuring the electronic impedance of sensor electrodes in the 16-well E-Plates using a parameter-termed cell index $(\mathrm{CI})$. In this RT-CES system, a $10-\mathrm{mV} \mathrm{AC}$ voltage at three different frequencies $(10 \mathrm{kHz}, 25 \mathrm{kHz}$, and $50 \mathrm{kHz})$ was applied to the microelectrodes for $\sim 0.1 \mathrm{~s}$, and the electrical current was monitored. The impedance of the system is determined by the ratio of the applied voltage and the response current. The cell index (CI) was calculated according to the following equation (Solly et al. 2004):

$$
C I=\max _{i=1, \ldots N}\left(\frac{R_{\text {cell }}\left(f_{i}\right)}{R_{0}\left(f_{i}\right)}-1\right)
$$

where $N$ is the number of the frequency points at which the impedance is measured (in this system, $N=3$ for $10 \mathrm{kHz}, 25 \mathrm{kHz}$, and $50 \mathrm{kHz})$, and $R_{0}(f)$ and $R_{\text {cell }}(f)$ are the electrode resistance/impedance without cells and with cells at each individual frequency, respectively.

$\mathrm{CI}$ is able to provide quantitative information about the cell status, including cell number, viability, and morphology. With a certain number of cells on the electrodes, changes in cell activities will lead to a change in cell index. For example, cell proliferation on the electrodes will result in an increase in $R_{\text {cell }}(f)$, and thus, an increase in cell index. On the other hand, drug-induced 
cell detachment, cell death, or apoptosis will result in a reduced $R_{\text {cell }}(f)$ and therefore a reduced cell index.

\section{Cell culture}

The prostate cancer cell lines $\mathrm{LNCaP}$ clone FGC $\left(\mathrm{ATCC}^{\bullet}\right.$ CRL-1740), DU 145(ATCC ${ }^{\circ} \mathrm{HTB}-81$ ), and PC-3 (ATCC ${ }^{\circ}$ CRL-1435) were cultured in RPMI (Hyclone, Logan, UT) supplemented with 10\% FBS (Hyclone) and antibiotics (100 IU/mL penicillin and $100 \mu \mathrm{g} / \mathrm{mL}$ streptomycin) (Hyclone). The Primary Prostate Epithelial Cells, Normal, Human (ATCC • PCS-440-010) (purchased 10/07/15), were cultured in Prostate Epithelial Cell Basal Media (ATCC $^{\bullet}$ PCS-440-030) supplemented with Prostate Epithelial Cell Growth Kit components (ATCC PCS-440-040) and Gentamicin-Amphotericin B Solution (ATCC ${ }^{\bullet}$ PCS-000-025).

Cells were cultured in $75 \mathrm{~cm}^{2}$ flask and were incubated at $37{ }^{\circ} \mathrm{C}$ in an atmosphere of $5 \% \mathrm{CO}_{2}$ in air. The medium was renewed every 2-3 days. When $80-90 \%$ confluent, cells were detached from the flask using $0.25 \%$ trypsin with $0.53 \mathrm{mM}$ EDTA (Lonza, Walkersville, MD) solution. Cells were centrifuged to remove trypsin and were suspended in fresh Prostate Epithelial Basal Medium or RPMI medium. Cell number in the suspension was determined using the Vi-cell XR cell counting system (Beckman Coulter, Miami, FL). Desired cell concentrations were obtained by diluting the cell suspension for further experiments.

\section{Drug treatments}

Docetaxel and sunitinib malate were obtained from BIOTANG, Inc. (Waltham, MA, USA). Abiraterone acetate was obtained from AK Scientific (Union City, CA, USA). Mitoxantrone was obtained from Selleck Chemical. Carboplatin was obtained from AdipoGen Life Sciences (San Diego, CA, USA). All were purchased through Fisher Scientific.

To make stock solutions, carboplatin was dissolved in deionized (DI) $\mathrm{H}_{2} \mathrm{O}$ to make a $10-\mathrm{mg} / \mathrm{mL}$ stock solution. Docetaxel, sunitinib malate, abiraterone acetate, and mitoxantrone were dissolved in DMSO at $1 \mathrm{mg} / \mathrm{mL}$, $40 \mathrm{mg} / \mathrm{mL}, 10 \mathrm{mg} / \mathrm{mL}$, and $40 \mathrm{mg} / \mathrm{mL}$, respectively, to make stock solutions that were further diluted with media for treatments.

For traditional optical-based cell viability assays, cells were plated onto 96-well plates using a Multidrop 384 (Thermo Electron Corporation) at 5000 cells per well (cpw) for DU 145, $7000 \mathrm{cpw}$ for PC-3, 17,000 cpw for LNCaP, or $8000 \mathrm{cpw}$ for normal prostate and were allowed to grow for $48 \mathrm{~h}$. Cells were treated with docetaxel, abiraterone acetate, sunitinib malate, mitoxantrone, and carboplatin or an equivalent concentration of the diluted vehicle at the concentrations indicated in figure legends. Addition of drugs was facilitated by the use of a HP
D300e Digital Dispenser (Hewlett-Packard) and Biomek NX MC compound dispenser (Beckman Coulter, Indianapolis, IN, USA). Cells were treated with drug for 24 and $48 \mathrm{~h}$, after which cell viability assays were performed.

\section{Cell viability assays}

Cell viability was assessed using the CellTiter $96^{\circ}$ Non-Radioactive Cell Proliferation Assay (Promega, Madison, WI, USA) and resazurin assays. The CellTiter $96^{\circ}$ Non-Radioactive Cell Proliferation Assay is a homogenous 3-[4, 5-dimethylthiazol-2-yl]-2, 5-diphenyltetrazolium salt solution assay, more commonly known as MTT. Briefly, $15 \mu \mathrm{L}$ of the MTT dye was added to each well and the plate returned to the incubator. Live cells converted the MTT to an insoluble product, formazin. After $3 \mathrm{~h}$, $100 \mu \mathrm{L} /$ well solubilization solution was added. Cell viability was determined by reading absorbance at $570 \mathrm{~nm}$ once all of the formazin crystals were dissolved. The absorbance signal was quantified using a SpectraMax M5 plate reader (molecular devices) equipped with SoftMax Pro software.

Resazurin assay indicated mitochondrial metabolic activity in live cells. Briefly, $10 \mu \mathrm{L}$ of $1 \mathrm{mg} / \mathrm{mL}$ resazurin (Fisher Scientific/Acros Organics, Fair Lawn, NJ, USA) dissolved in sterile $\mathrm{DI} \mathrm{H}_{2} \mathrm{O}$ was added to each well. The plates were returned to the incubator for $3 \mathrm{~h}$. The live cells reduced the resazurin to resorufin, a fluorescent product. The fluorescence was measured using a PHERAstar (BMG Labtech, Germany).

In both assays, the amount of product was proportional to the number of live cells. Viable cell percentage was calculated by dividing the background-subtracted mean absorbance of treated sample by the background-subtracted mean untreated sample of that plate, then multiplying by 100 .

$\begin{aligned} \% \text { viable cells } & =[(\text { average treated })-(\text { average background })] \\ & /[(\text { average untreated })-(\text { average background })] \times 100 .\end{aligned}$

\section{Impedance-based RTCA measurement}

To run an experiment, each 16-well E-plate was prepared with $50 \mu \mathrm{L}$ of media per well and then placed into the analyzer to get a baseline reading of the impedance-based cell index signal. After the baseline was established, the E-plate was removed from the analyzer and was seeded with the same concentration of cells per well for each cell line as the optical assays. Cell volume for each well was in $100 \mu \mathrm{L}$ of media. After cell plating, E plate was set in the cell culture hood for $30 \mathrm{~min}$ at room temperature to ensure the cells were settled down to the microelectrodes on the bottom surface. The E-plate was then placed back to the analyzer in the incubator. The monitoring of impedance-based CI signal was set up through the software. Data was 
collected every $30 \mathrm{~min}$ for $96 \mathrm{~h}$ for cell growth measurement. For drug treatment tests, $\mathrm{CI}$ data was recorded for approximately $48 \mathrm{~h}$ for cell growth, then the instrument was paused, and the cells were treated with desired concentrations of docetaxel, abiraterone acetate, sunitinib malate, mitoxantrone, carboplatin, and equivalent concentrations of the diluted vehicle at given concentrations as controls. Drug treatment was a $10 \times$ final concentration, and the volume was $1 / 10$ volume of the culture volume in the wells, i.e., $15 \mu \mathrm{L}$ in each well. After drug treatment, CI data was continuously recorded for another $48 \mathrm{~h}$ or otherwise stated in figure legends.

\section{Results and discussion}

\section{Real-time monitoring of the growth characteristics for} multiple prostate cancer cell lines

By culturing biological cells on the electrode surface, the technique can directly sense detailed information about cellular activities occurring on the electrode surface by measuring the induced capacitance and/or resistance changes, eliminating multiple labeling and amplification steps typically used in many other cell-based methods. In addition, this can be done in a medium throughput platform and this is especially useful when dealing with a number of different cell lines. Figure 1 shows a group of growth curves of three different prostate cancer cell lines (DU145, LNCaP, PC-3) along with the normal prostate cell line, obtained by the impedance-sensing technique. As shown in Fig. 1, the recorded impedance-based cell growth curves clearly distinguished the growth characteristic/profile of these different prostate cancer cells. It is believed that these growth curves mainly reflected the specific degree of adhesion of these cells during the entire course of interaction with the electrodes, as well as the cell proliferation rate on the electrode. A correlation between the impedance-based cell index and the cell number for each cell line is demonstrated in Additional file 1: Figure S1. Among the cell lines, DU145 is derived from a brain metastasis of the prostate and does not express PSA. Its doubling time is approximately $29 \mathrm{~h}$. In Fig. 1, it showed the highest impedance-based cell index during the cell proliferation and stationary phase. LNCaP is derived from a needle biopsy of the subclavicular lymph node and is androgen sensitive and still expresses PSA. Its doubling time is approximately $60 \mathrm{~h}$, and it is generally regarded as poorly adhesive to cell culture substrate. The growth curve of LNCaP showed the lowest cell index among all cell lines at all stages. PC-3 is derived from a lumbar vertebrae metastasis, and it is androgen insensitive and does not express PSA. Its doubling time is $24-40 \mathrm{~h}$ varied in different conditions or laboratories. In this test, its growth curves showed moderate impedance-based cell index at all stages compared to DU145 and LNCaP cells. The growth of normal prostate epithelial cell line PCS-440-010 on the electrodes generated the impedance-based signal that was in between those tested prostate cancer cell lines. The results demonstrated that each cell line exhibited a unique impedance profile for its attachment and proliferation on the electrodes, highlighting the ability of the electrical impedance cell-based technique to identify the different adhesion properties and growth rates of the various cell lines. The different adhesion properties of the cells to the electrode are explained by the bonding forces between the plasma membrane and electrode as well as the specific combination of the surface molecules of each cell line (Asphahani and Zhang 2007). To extend, the results also suggest that this technique should be able to distinguish the growth characteristics of prostate cancer cells at different stages or different types.

\section{Rapid selection of effective anti-cancer drugs to a certain type of prostate cancer cell line}

In clinic, multiple anticancer drugs are available for treatment of cancers. However, cancer patients with different types of cancer or at different stages of a cancer generally respond to each drug with varying efficacy.

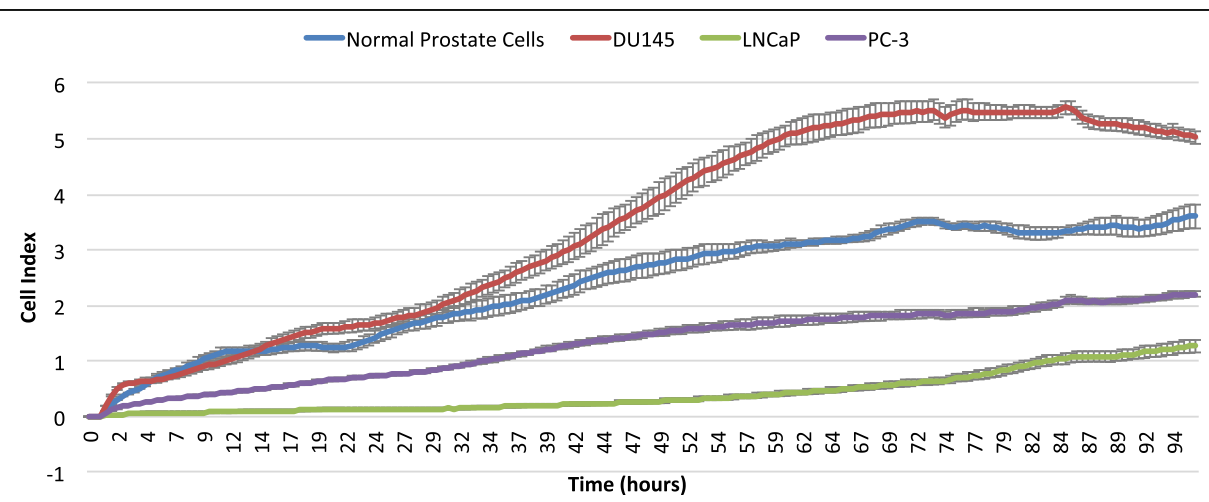

Fig. 1 Proliferation curves of prostate cell lines. ECIS chart showing proliferation measurement of three metastatic prostate cancer cell lines--DU145, $P C-3$, and LNCaP and the normal prostate cell line. Error bars represent standard error of $n=4$ values 
The heterogeneity of the population contributes to different responses to drugs of patients with seemingly same cancer and same stage due to the fact that each tumor is as unique as that person. The current standard of care for mCRPC is treatment with hormone therapy until the cancer becomes androgen independent. Once a cancer is identified as androgen independent, the patient is switched to another chemotherapeutic, the primary drug of choice is docetaxel (Antonarakis and Armstrong 2011; Flaig et al. 2007). A rapid and easy-to-use screening method for selecting an effective drug for a patient would be most beneficial to the treated patient. Here, we demonstrated the proof-of-concept of the use of impedance-sensing technique for such purpose.

Using LNCaP cell line, Fig. 2 shows the cellular responses to four anticancer drugs with respective dose ranges obtained by the impedance technique. These chemotherapy drugs were selected from the American Cancer Society and the National Cancer Institute lists of drugs used in treatment of prostate cancer. Each drug works on the cancer cells via a different mechanism of action. Docetaxel works by binding the $\beta$ subunits of tubulin in microtubules, thereby stabilizing them, preventing the de-polymerization required for mitosis, which induces cell apoptosis. Abiraterone acetate acts as an irreversible

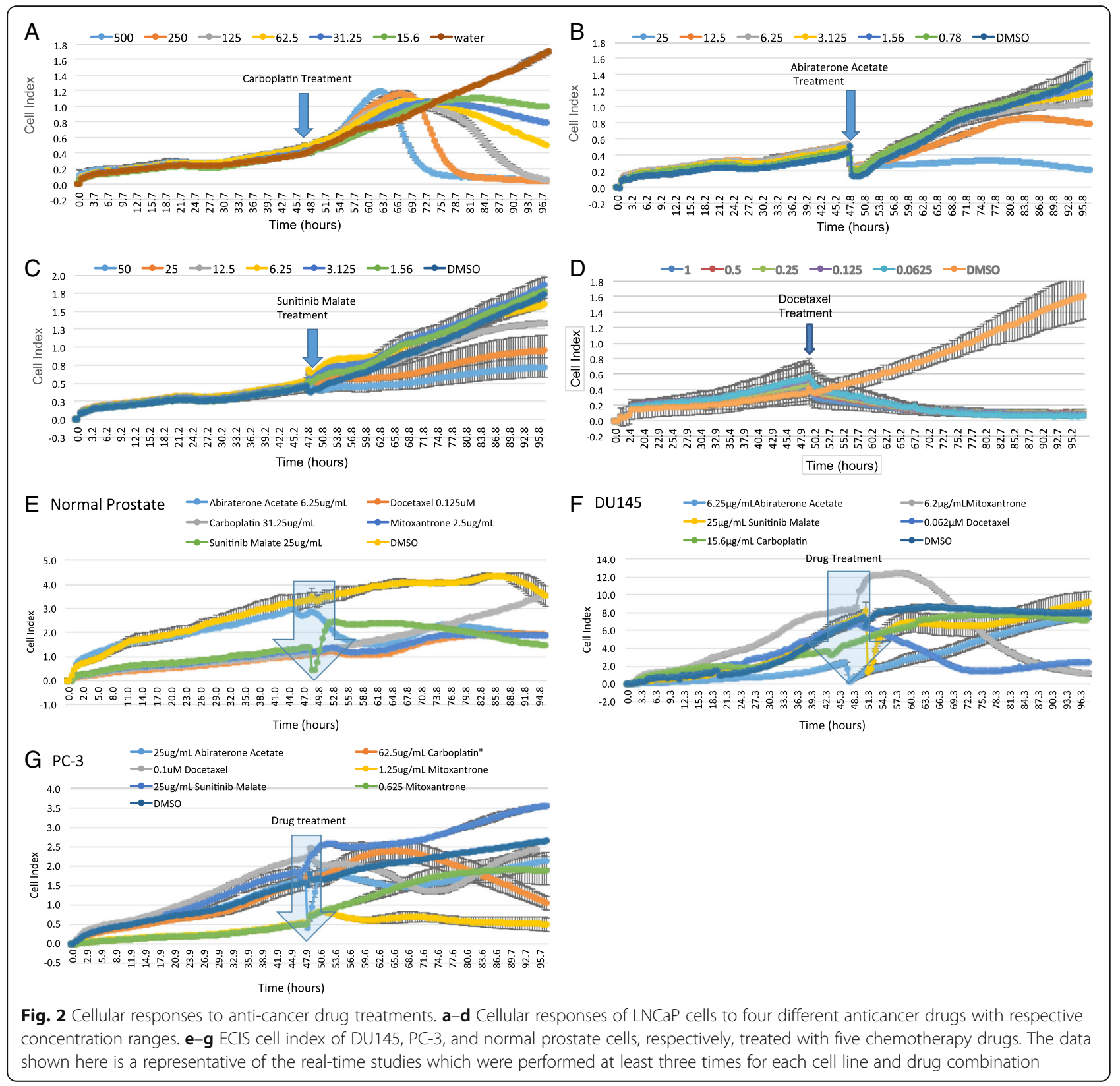


inhibitor of CYP17A1 leading to a significant loss of androgen production in the peripheral organs, particularly adrenal androgens. Sunitinib malate inhibits multiple receptor tyrosine kinases (RTKs) such as vascular endothelial growth factor receptors (VEGFR1, VEGFR2, and VEGFR3) and stem cell factor receptor (KIT). Mitoxantrone causes DNA fragmentation and inhibits topoisomerase II. Mitoxantrone was one of the first drugs approved to treat mCRPC (Antonarakis and Armstrong 2011; Hwang 2012). Carboplatin undergoes activation inside cells and forms reactive platinum complexes that cause the intra- and inter-strand cross-linkage of DNA molecules within the cell. As shown in Fig. 2, treatment with each drug to LNCaP cells generated a distinguished response curve. Docetaxel at its effective doses caused a gradual continuously decreasing cell index. Carboplatin at its effective doses caused a quite rapid increase in cell index to a peak immediately after the treatment, followed by a sharp decrease until all the cells died. Abiraterone acetate at its effective doses induced an immediate steep drop of cell index, followed by a gradual increase of cell index to a flat peak, and then a slow decrease until all the cells died. Sunitinib malate induced a small gentle peak of cell index at time of drug treatment and continued its increase trend with a slower rate; it seemed that sunitinib malate did not cause cell death but only slowed down cell proliferation.

We also examined the responses of other cell lines to these drugs. Figure 2e shows the response of DU145 cell line to the five drugs at a given effective dose. Again, the cellular responses were distinct, reflecting the different effect of the drug action onto the cells on the electrodes.

It is believed that such signal profile is mainly a result of drug effect on the cellular contacts with the electrodes. Generally, when cells adhere onto the electrode surface, although cell membranes are usually $\sim 10$ $100 \mathrm{~nm}$ away from the electrode surface, the electrodes are still able to sense the bottom portion of cells. Cell adhesion to a substrate occurs through three major contacts including focal contacts, close contacts, and extracellular matrix contacts, and each of them has its specific separation distance from the substrate. Upon external stimuli, cells commonly experience dynamic relocation or rearrangement of certain cellular contents; some of these result in changes in cell adhesion degree, membrane ruffling, and activation of receptors at the cell surface or receptor endocytosis (Lu et al. 2001; Milligan 2003). Those which occur at the bottom portion of the cells and affect the contacts between the cells and the electrode can be sensed by the electrodes, manifested by the impedance-based cell index signal, such signal is equivalent to dynamic mass redistribution (DMR), a signal previously reported in label-free cell-based optical biosensors (Fang 2006; Fang et al. 2005a; Fang et al. 2006; Fang et al. 2005b). The DMR signal is a sum of all mass redistribution within the sensible region induced by the stimuli, although it is not specific for individual events/pathways, it is distinctive in overall profile of different cell lines in response to a same drug or the same cell line in response to different stimuli (e.g., drug treatment), as shown in Fig. 2.

Besides the capacity to generate the characteristic profile for each drug, these response curves also provide some dynamic and kinetic information of the drugs to the tested cells. For example, in Fig. 2, docetaxel caused an immediate effect on the cells that was manifested by the decreasing signal immediately after the treatment, and drug effect reached the maximal (signal decreased to baseline) after approximately $24 \mathrm{~h}$. With a different action mechanism, carboplatin did not exhibit effect on the cells within the first $\sim 16 \mathrm{~h}$ after treatment. At high concentrations, drug effect can be detected at $\sim 24 \mathrm{~h}$, but with lower concentrations; the effect of this drug was best observed at $\sim 48 \mathrm{~h}$ after treatment, exhibiting a dose-dependent manner. For abiraterone acetate, only at the highest tested concentration, the drug effect on the cells (signal decrease) can be immediately seen after the treatment; the effect of moderate to low concentration of this drug can be observed after $36 \mathrm{~h}$ of treatment. Sunitinib malate exhibited a cytostatic effect on the cells soon after the treatment (within $8 \mathrm{~h}$ ); it seemed that the drug did not cause cell death, and it more likely just slowed down the cell proliferation (the signal still kept increasing but with a slower rate). Commonly used cell viability assays can only provide cell information about cell viability at a given time point, usually at 24 or $48 \mathrm{~h}$ or other defined time, but would never be possible to provide such real-time dynamic and kinetic information about the cellular responses to a drug during the entire course of the treatment. Other cell lines showed similar drug curve profiles; however, cellular response varied by cell line (data not shown).

\section{Correlations between the electric impedance-based analysis and cell viability assays}

We further looked into the results of impedance technique and extracted data at a given time point (for example at $24 \mathrm{~h}$ or $48 \mathrm{~h}$ after treatment) to generate dose response curves, which were compared to those obtained by the traditional cell viability MTT or resazurin assays at the same time point. Figure 3 shows the dose response curves of $\mathrm{LNCaP}$ cells in response to docetaxel, carboplatin, abiraterone acetate, and sunitinib malate extracted from the growth curves in Fig. 2 at either 24 or $48 \mathrm{~h}$, along with the dose response curves that were obtained by the traditional MTT or resazurin cell viability assay at the same time points. It should be noted that the $y$-axis is the percentage of the signals of treated 


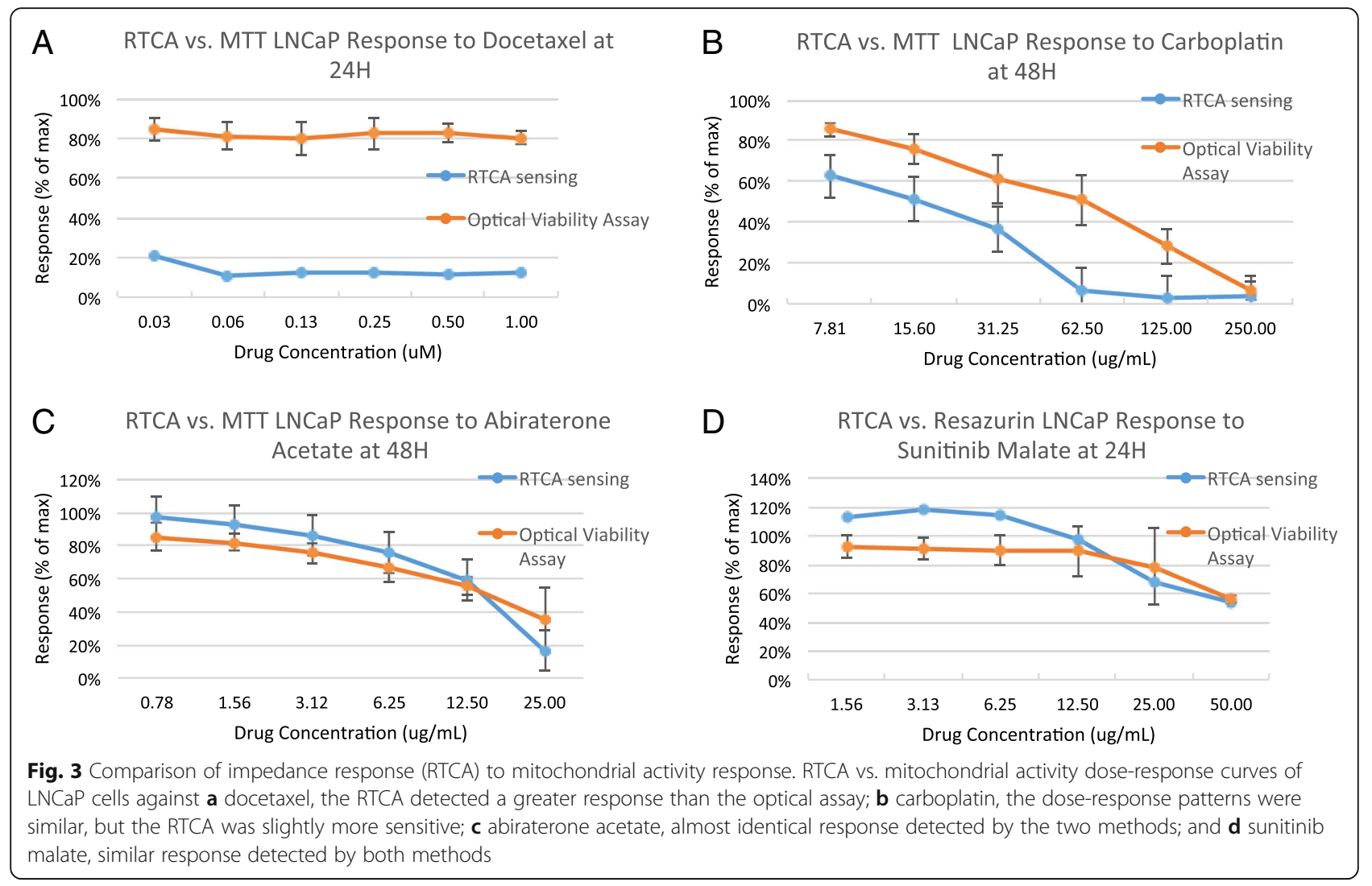

samples compared to the untreated controls, which would be a different meaning in the RTCA method and cell viability assay, but in both cases, it reflects the effect of a drug treatment. More specifically, in cell viability assay, it represents the percentage of surviving cells after the drug treatment, while in RTCA system, it represents the percentage of the total adhesive interactions that the cells have with the electrodes after the drug treatment and measurable by impedance. As shown in Fig. 3a, all the dose response curves from the RTCA system present similar trends as those from the cell viability assays. The percentage signals from the two methods are reasonably close in the responses to three drugs (carboplatin, abiraterone acetate, and sunitinib malate) except one drug (doxcetaxel).

It is noted that although the dose response curve of LNCaP cells to docetaxel from the RTCA system presents a similarly trend to that from the cell viability assays; however, the magnitude in the percentage signal between the two methods were largely different, with much lower percentage signal measured by RTCA but still high percentage in cell viability. Knowing that the meaning of the percentage signals are different in the two approaches, it is understandable and explainable for the big difference. The difference in the percentage signal between the two approaches is related to the effect/action mechanism of the drug on the cells, which may be better measurable by one than the other. In the case of docetaxel, it is known to cause apoptosis which has an effect on membrane integrity and cell morphology. Cells are reported to shrink and become round due to cytoskeletal protein digestion by caspases. Figure 4 shows the microscopic images of LNCaP cells after docetaxel treatment, showing the round cells but most likely still viable. Once the cells became round, they were less adhesive to the electrodes, which was well measured by the RTCA system (Leung et al. 2005), while the MTT cell viable assay was not able to detect the changes in the cells adhesion but only detect the viability of cells. For other tested drugs, they likely affect cell adhesive interactions with the electrodes at a similar extent as to the cell viability, which was manifested by the two similar and close dose response curves in each plot in Fig. 3. It is also worthy of note that the RTCA makes it easier to visualize the slower entry of carboplatin into the cells than the other drugs. This slow entry is responsible for the observed effects at a delayed time of 20 to $26 \mathrm{~h}$ after treatment. These comparisons indicated the advantages of the impedance-based RTCA method lie in its capability for sensitive and real-time monitoring the cellular responses, including not only cell viability but also cell adherence and other subtle cell morphology changes induced by drug treatments. Such ability is especially useful to detect cell responses at early stage of drug 

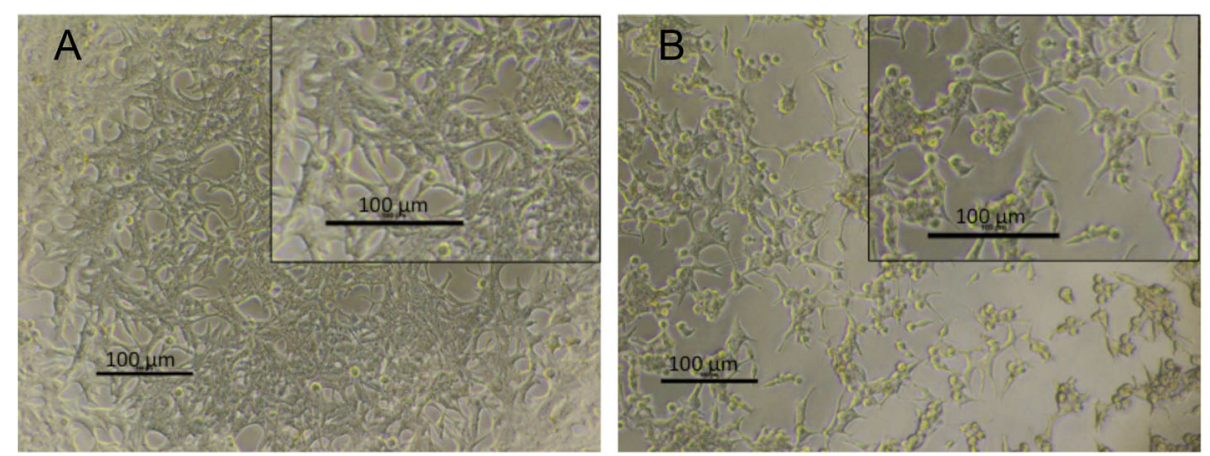

Fig. 4 Bright field images of LNCaP response to $0.01 \mu \mathrm{M}$ docetaxel. a LNCaP cells treated with DMSO control. b LNCaP cells treated with $0.01 \mu \mathrm{M}$ docetaxel for $48 \mathrm{~h}$. Notice the smaller and more rounded shape of the treated cells. The inserts are a part of each image with a higher magnification. Scale bars are $100 \mu \mathrm{m}$

treatment, while it is generally not possible for traditional MTT assay to detect.

\section{Determining the effectiveness of a drug to different types of cell lines}

With a different experimental design, evaluation of a drug to a number of cell lines can be performed, and real-time data can be obtained. This platform would be useful when applications of a newly available drug need to be tested on different stages of a cancer, or on different types of cancers. For prostate cancer, chemotherapy is typically used once the cancer has become androgen independent and spread outside the prostate gland; in most cases, the first chemo drug given is docetaxel (Antonarakis and Armstrong 2011). Figure 5 shows the response curves of different prostate cell lines (DU145, LNCaP, and PC-3) along with the normal prostate cell line in response to docetaxel. As demonstrated, docetaxel is effective for treatment to DU145 and LNCaP, but has little effect on the normal prostate cancer cell. However, it shows initial effect on PC-3 cells, but interestingly, PC-3 cells exhibit a compensatory response, meaning some pre-existing mechanism that is triggered upon exposure to docetaxel. Studies show docetaxel is effective in approximately $50 \%$ of cases, and most patients develop resistance to this chemotherapeutic (Hwang 2012; Liu et al. 2013).

\section{Applications for special tests--drug resistance and combination treatments}

This technique also allows examination of the effect of consecutive/repeated treatment with drug. Figure 6 shows the response curves of PC-3 cell line to repeated treatments with $0.1 \mu \mathrm{M}$ docetaxel. The repeated treatments with docetaxel did not enhance the effect of docetaxel on PC-3 cells, meaning that PC-3 cells exhibited permanent response to docetaxel after the first treatment. This would be a useful tool for a clinic office to
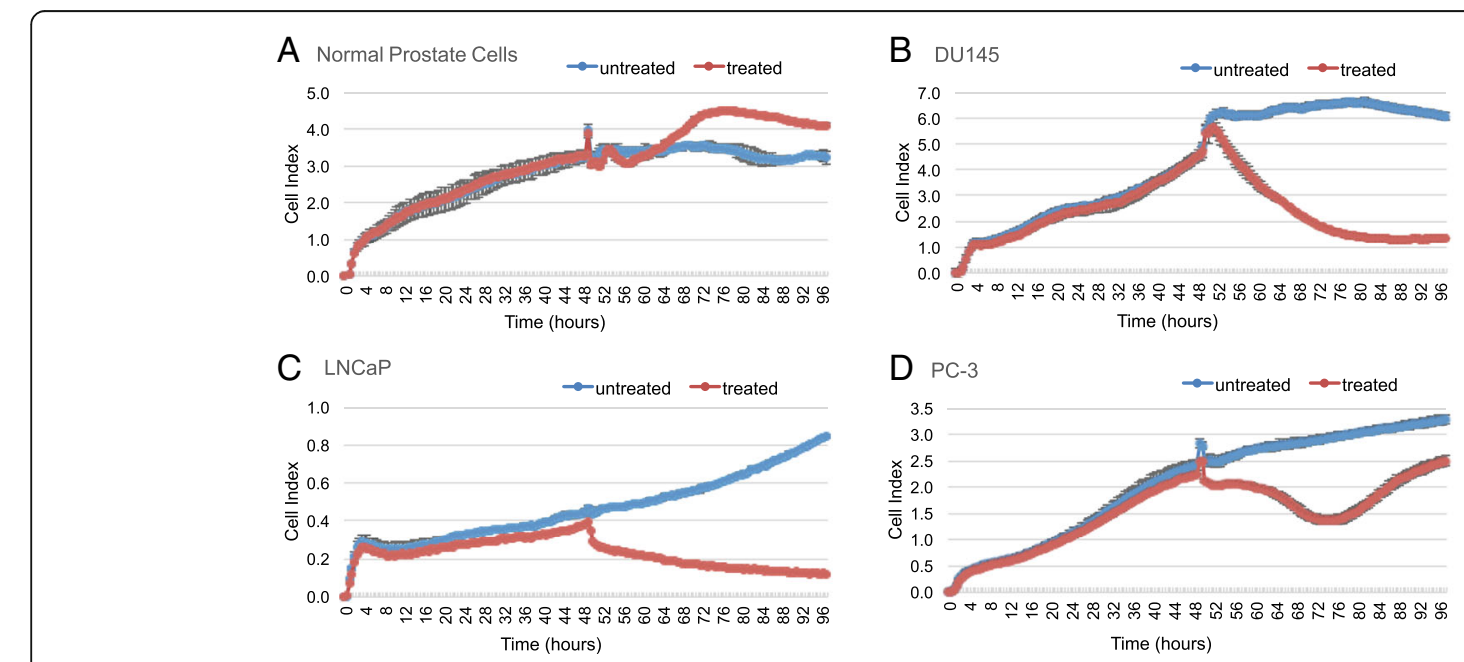

Fig. 5 Comparison of four cell lines' impedance response to docetaxel. The response of the four cell lines treated with $0.1 \mu \mathrm{M}$ docetaxel compared to DMSO control 


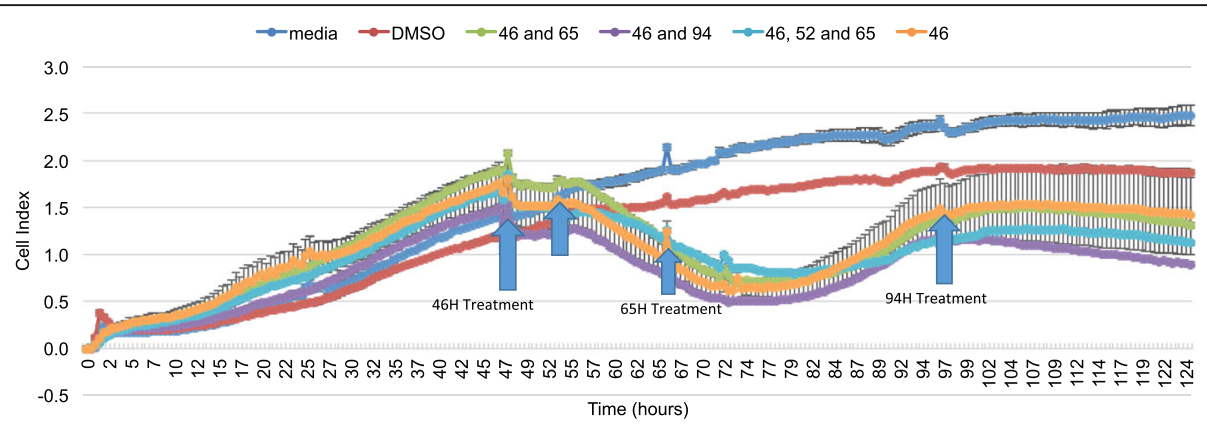

Fig. 6 PC-3 impedance response to repeated docetaxel treatments. Cell index curve of PC-3 cells with repeated treatments of $0.1 \mu M$ docetaxel compared to controls. Each spike represents a pause in the experiment for drug treatment to at least one set of wells on the plate

quickly rule out those drugs that may not be effective in a patient due to compensatory response.

The other useful application of RT-CES is to test the effect of combination of chemotherapy drugs. Although chemo drugs can be used one at a time, more often, a combination of drugs may provide higher efficacy to a patient if appropriate combination can be found. It is recommended to use drugs with different mechanisms of action when treating with drug combinations (Tsakalozou et al. 2012). For this combination drug study, we selected the kinase inhibitor, sunitinib malate, and the microtubule stabilizing agent docetaxel. With this regard, we demonstrated the tests of DU145 cells treated with a combination of sunitinib malate and docetaxel (Fig. 7). The single drug treatment of $5 \mu \mathrm{g} / \mathrm{mL}$ sunitinib malate to DU145 cells did not result in a reduction in $\mathrm{CI}$; this dose did not even exact the cytostatic effect that was observed at higher monotherapy sunitinib malate doses. A reduction in CI was seen when the cells were treated with $10 \mathrm{nM}$ docetaxel as a single drug, but reduction did not decrease to baseline and appeared to plateau out. This plateau was likely due to that all of the drugs had been utilized by the cells in the well or the cells stopped taking it in as the equilibrium had been achieved between cells and media. Higher doses of docetaxel (100 nM) decreased CI signal to background. When $5 \mu \mathrm{g} / \mathrm{mL}$ of sunitinib malate was combined with lower doses of docetaxel a reduction in CI was observed in all doses. The same sharp immediate decrease in CI signal was observed for the combined treatment wells as the single-drug docetaxel except that the CI signal was reduced to baseline. These combination results are supported by the optical absorbance and fluorescence assay findings that were completed simultaneously with the RTCA experiment, data not shown. This reduction in signal was not seen with all combinations of these two drugs on DU145 cells.

It is important to note that all cell lines tested did not draw us to the same conclusion for the drug combination. The reduction in $\mathrm{CI}$ for the $\mathrm{PC}-3$ cell line was greater in the presence of the combined drugs, and the lag between reduction and increase was longer. This

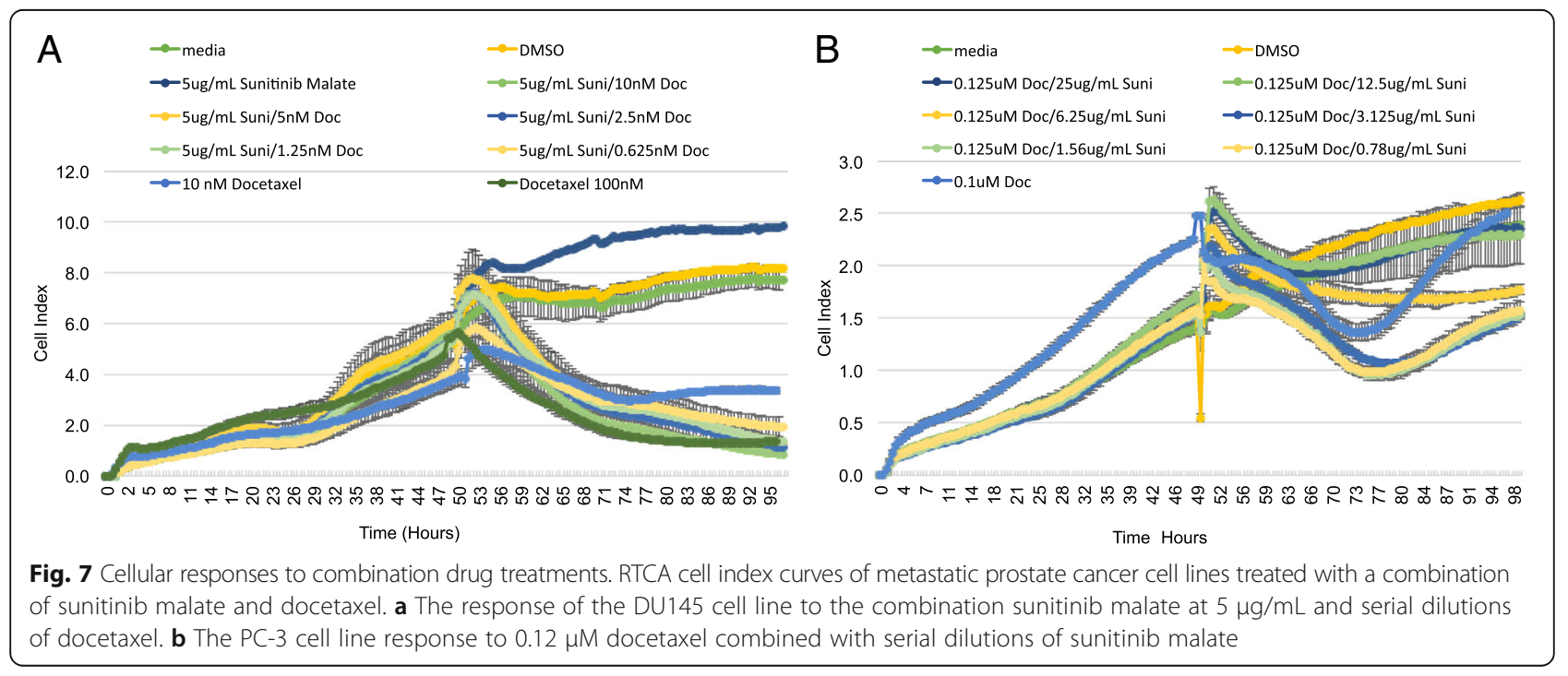


indicated that there was an increase in toxicity by the drug combination in PC-3 cells; however, the increased toxicity was not sustainable. PC-3 cell line was still resistant to docetaxel. These results demonstrated that the RTCA can be used to identify drug combinations that can effectively be used to treat prostate cancer.

\section{Conclusions}

Using several established metastatic prostate cell lines and common chemotherapeutic drugs for prostate cancer, this study demonstrated the proof-of-concept of the real-time impedance method for potential application toward selection of personalized chemotherapy drugs for patients. Such impedance-based technique measured the drug-induced effect on cellular adhesion, proliferation, and other changes of the cells cultured on the electrodes. Agreement of overall trend in drug responses obtained by this impedance-based method and the traditional optical-based cell viability assay was demonstrated. Through experimental design, RT-CES can be applied to a certain type of cancer cell line against a panel of chemotherapy drugs before the patient receives the drug treatments. It can also rule out ineffective drugs against a particular tumor or the drug that may develop resistance by a patient. Certainly, such concept needs to be applied to patient-derived samples of known drug response to validate its ability to predict the chemotherapy outcome before actual application. In drug screening, this label-free real-time screening method could benefit in minimizing some limitations in dye conversion-based end point analysis in traditional optical-based cell viability assays.

\section{Additional file}

Additional file 1: Figure S1. Prostate cell titration at $24 \mathrm{~h}$ post seeding. Cell dilution series showing impedance linear relationship to cell number $24 \mathrm{~h}$ after plating. Changes in impedance reflect change in cell number. (PNG $55 \mathrm{~kb}$ )

\footnotetext{
Abbreviations

ATCC : American Type Culture Collection; Cl: Cell index; cpw: Cells per well; CYP17A1: Member of the cytochrome P450 superfamily of enzymes, catalyzes many reactions involved in drug metabolism and synthesis of cholesterol, steroids, and other lipids; $\mathrm{DI} \mathrm{H}_{2} \mathrm{O}$ : Deionized water; DMR: Dynamic mass redistribution; DNA: Deoxyribonucleic acid EDTA: Ethylenediaminetetraacetic acid; FBS: Fetal bovine serum; H: Hours; KIT: Stem cell factor receptor; MCRPC: Metastatic castration-resistant prostate cancer; MTT: 3-[4, 5-Dimethylthiazol-2-yl]-2, 5-diphenyltetrazolium salt; N: The number of the frequency points; PSA: Prostate specific antigen, protein produced by prostate gland, high levels associated with prostate cancer; $R_{0}(f)$ : Electrode resistance/impedance at initial frequency without cells; $R_{\text {cell }}(f)$ : Electrode resistance/impedance at each individual frequency with cells; RTCA DP: Real-time cell analyzer dual plate; RTCA: Real-time cell analyzer; RT-CES: Real-time cell electronic sensing; RTKs: Receptor tyrosine kinases; VEGFR1, VEGFR2, and VEGFR3: Vascular endothelial growth factor receptors; $\beta$ subunits: Beta subunits, microtubules are composed of alpha and beta tubulin subunits
}

\section{Acknowledgements}

The authors thank Ginger R. Smith of the NCCU BRITE high throughput screening (HTS) lab for assistance with the HP D300e Digital Dispenser and Biomek NX MC compound dispenser.

\section{Funding}

This research was partially supported by NSF grant CBET no. 1159871.

\section{Availability of data and materials}

The dataset supporting the conclusions of this article are included within the article (and its additional file).

\section{Authors' contributions}

AFA designed the experiments, performed the data analysis, reviewed the literature, and drafted the manuscript. COA performed the experiments and assisted with the data analysis. LY reviewed the literature and provided guidance in designing, writing, and revising the manuscript. All authors read and approved final manuscript provided guidance.

\section{Competing interests}

The authors declare that they have no competing interests.

\section{Publisher's Note}

Springer Nature remains neutral with regard to jurisdictional claims in published maps and institutional affiliations.

Received: 6 June 2018 Accepted: 1 August 2018

Published online: 18 August 2018

\section{References}

(ASCO), American Society of Clinical Oncology. (2017). What is personalized medicine. Cancer.Net. from https://http://www.cancer.net/navigating-cancercare/how-cancer-treated/personalized-and-targeted-therapies/whatpersonalized-cancer-medicine

Antonarakis ES, Armstrong AJ. Evolving standards in the treatment of docetaxelrefractory castration-resistant prostate cancer. Prostate Cancer Prostatic Dis. 2011;14(3):192.

Arias LR, Perry CA, Yang L. Real-time electrical impedance detection of cellular activities of oral cancer cells. Biosens Bioelectron. 2010;25(10):2225-31.

Asphahani F, Zhang M. Cellular impedance biosensors for drug screening and toxin detection. Analyst. 2007;132(9):835-41. https://doi.org/10.1039/ b704513a.

Atay S, Piskin K, Yilmaz F, Cakir C, Yavuz H, Denizli A. Quartz crystal microbalance based biosensors for detecting highly metastatic breast cancer cells via their transferrin receptors. Anal Methods. 2016;8(1):153-61. https://doi.org/10.1039/ c5ay02898a.

Braunhut SJ, Mclntosh D, Vorotnikova E, Zhou T, Marx KA. Detection of apoptosis and drug resistance of human breast cancer cells to taxane treatments using quartz crystal microbalance biosensor technology. Assay and Drug Development Technologies. 2005;3(1):77-88. https://doi. org/10.1089/adt.2005.3.77.

Chen J, Zhang J, Huang L, Lin X, Chen G. Hybridization biosensor using 2nitroacridone as electrochemical indicator for detection of short DNA species of chronic myelogenous leukemia. Biosens Bioelectron. 2008;24(3):349-55.

Cooper MA. Current biosensor technologies in drug discovery. Drug discovery. 2006. https://www.ddw-online.com/drug-discovery/p97058-currentbiosensortechnologies-in-drug-discovery-summer-06.html. Accessed on 8 June 2018.

Fang Y. Label-free cell-based assays with optical biosensors in drug discovery. Assay and Drug Development Technologies. 2006;4(5):583-95. https://doi. org/10.1089/adt.2006.4.583.

Fang Y. Label-free biosensors for cell biology. International Journal of Electrochemistry. 2011;2011 https://doi.org/10.4061/2011/460850.

Fang Y. Label-free drug discovery. Frontiers in Pharmacology. 2014;5:52. https:// doi.org/10.3389/Fphar.2014.00052.

Fang Y. Non-invasive optical biosensor for probing cell signaling. Sensors. 2007; 7(10):2316-29.

Fang Y, Ferrie AM, Fontaine NH, Yuen PK. Characteristics of dynamic mass redistribution of epidermal growth factor receptor signaling in living cells measured with label-free optical biosensors. Anal Chem. 2005a;77(17):5720-5.

Fang Y, Ferrie AM, Li G. Cellular functions of cholesterol probed with optical biosensors. Biochimica et Biophysica Acta (BBA)-Molecular Cell Research. 2006;1763(2):254-61. 
Fang Y, Li GG, Peng J. Optical biosensor provides insights for bradykinin B2 receptor signaling in A431 cells. FEBS Lett. 2005b;579(28):6365-74.

Flaig TW, Su L-J, Harrison G, Agarwal R, Glodé LM. Silibinin synergizes with mitoxantrone to inhibit cell growth and induce apoptosis in human prostate cancer cells. Int J Cancer. 2007;120(9):2028-33.

Giaever I, Keese CR. A morphological biosensor for mammalian cells. Nature. 1993;366(6455):591-2. https://doi.org/10.1038/366591a0.

Giaever I, Keese CR. Use of electric fields to monitor the dynamical aspect of cell behavior in tissue culture. IEEE Transactions on Biomedical Engineering. 1986; 33(2):242-7.

Hamburg MA, Collins FS. The path to personalized medicine. N Engl J Med. 2010; 363(4):301-4. https://doi.org/10.1056/NEJMp1006304.

Hu N, Zhou J, Su K, Zhang D, Xiao L, Wang T, Wang P. An integrated label-free cell-based biosensor for simultaneously monitoring of cellular physiology multiparameter in vitro. Biomed Microdevices. 2013;15(3):473-80. https://doi. org/10.1007/s10544-013-9747-y.

Hwang C. Overcoming docetaxel resistance in prostate cancer: a perspective review. Therapeutic advances in medical oncology. 2012;4(6):329-40.

Kaisti M. Detection principles of biological and chemical FET sensors. Biosens Bioelectron. 2017;98:437-48. https://doi.org/10.1016/j.bios.2017.07.010.

Kim HN, Bradford SA, Walker SL. Escherichia coli O157:H7 transport in saturated porous media: role of solution chemistry and surface macromolecules. Environmental Science \& Technology. 2009;43(12):4340-7. https://doi.org/10. 1021/es8026055.

Kloß D, Kurz R, Jahnke H-G, Fischer M, Rothermel A, Anderegg U, et al. Microcavity array (MCA)-based biosensor chip for functional drug screening of 3D tissue models. Biosens Bioelectron. 2008;23(10):1473-80.

Leung G, Tang HR, McGuinness R, Verdonk E, Michelotti JM, Liu VF. Cellular dielectric spectroscopy: a label-free technology for drug discovery. JALA: Journal of the Association for Laboratory Automation. 2005:10(4):258-69.

Linderholm P, Vannod J, Barrandon Y, Renaud P. Bipolar resistivity profiling of 3D tissue culture. Biosens Bioelectron. 2007;22(6):789-96.

Liu C, Zhu Y, Lou W, Nadiminty N, Chen X, Zhou Q, et al. Functional p53 determines docetaxel sensitivity in prostate cancer cells. Prostate. 2013;73(4):418-27.

Liu QJ, Wu CS, Cai H, Hu N, Zhou J, Wang P. Cell-based biosensors and their application in biomedicine. Chem Rev. 2014;114(12):6423-61. https://doi.org/ $10.1021 /$ cr2003129

Liu Q, Yu J, Xiao L, Tang JCO, Zhang Y, Wang P, Yang M. Impedance studies of bio-behavior and chemosensitivity of cancer cells by micro-electrode arrays. Biosens Bioelectron. 2009;24(5):1305-10.

Lu Z, Jiang G, Blume-Jensen P, Hunter T. Epidermal growth factor-induced tumor cell invasion and metastasis initiated by dephosphorylation and downregulation of focal adhesion kinase. Mol Cell Biol. 2001;21(12):4016-31.

McGuinness R. Impedance-based cellular assay technologies: recent advances, future promise. Curr Opin Pharmacol. 2007;7(5):535-40.

Milligan G. High-content assays for ligand regulation of G-protein-coupled receptors. Drug Discov Today. 2003;8(13):579-85.

Ramsden JJ, Li S-Y, Heinzle E, Prenosil JE. Optical method for measurement of number and shape of attached cells in real time. Cytometry Part A. 1995; 19(2):97-102.

Solly K, Wang X, Xu X, Strulovici B, Zheng W. Application of real-time cell electronic sensing (RT-CES) technology to cell-based assays. Assay and drug development technologies. 2004;2(4):363-72.

Tsakalozou E, Eckman AM, Bae Y. Combination effects of docetaxel and doxorubicin in hormone-refractory prostate cancer cells. Biochemistry Research International. 2012;2012:10. Article ID 832059. https://doi.org/10. 1155/2012/832059

Xiao C, Luong JHT. On-line monitoring of cell growth and cytotoxicity using electric cell-substrate impedance sensing (ECIS). Biotechnol Prog. 2003;19(3):1000-5.

Yang L, Arias LR, Lane TS, Yancey MD, Mamouni J. Real-time electrical impedance-based measurement to distinguish oral cancer cells and noncancer oral epithelial cells. Anal Bioanal Chem. 2011;399(5):1823-33.

Zhang Z, Guan N, Li T, Mais DE, Wang M. Quality control of cell-based highthroughput drug screening. Acta Pharm Sin B. 2012;2(5):429-38.

Zhou T, Marx KA, Warren M, Schulze H, Braunhut SJ. The quartz crystal microbalance as a continuous monitoring tool for the study of endothelial cell surface attachment and growth. Biotechnology Progress. 2000;16(2):26877. https://doi.org/10.1021/Bp000003f.

\section{Submit your manuscript to a SpringerOpen ${ }^{\circ}$ journal and benefit from:}

- Convenient online submission

- Rigorous peer review

- Open access: articles freely available online

- High visibility within the field

- Retaining the copyright to your article

Submit your next manuscript at $\boldsymbol{\nabla}$ springeropen.com 